\title{
KERNEL FUNCTIONS AND NUCLEAR SPACES
}

\author{
BY JOSEPH WLOKA
}

Communicated by A. E. Taylor, May 7, 1965

As is well known, it is possible to represent any complete, locally convex space $E$ as

$$
E=\underset{\leftarrow \alpha \in A}{\operatorname{proj}} E_{\alpha}
$$

where the $E_{\alpha}$ are Banach spaces. If the projective mappings of (1) are nuclear [2], $E$ is called a nuclear space. For complete spaces, this definition is equivalent to Grothendieck's original one (see [2], [8]). It is possible to treat nuclearity for countable inductive limit spaces in a dual fashion:

Definition 1. $E$ is called an (LN)-space if

(1) $E=$ ind $_{\rightarrow n} E_{n}, n=1,2, \cdots$, where the $E_{n}$ are Banach spaces,

(2) the inductive mappings (imbeddings) are nuclear.

We have the following theorem.

THEOREM 1. Every (LN)-space is nuclear (in the sense of Grothendieck).

For regular inductive limit spaces the inverse theorem is also true (a space $E=$ ind $_{\rightarrow n} E_{n}$ is called regular, if every bounded set $A \subset E$ is already bounded in some $E_{n_{0}}$ ).

THEOREM 2. If the regular space $E=$ ind $_{\rightarrow n} E_{n}$, the $E_{n}$ being Banach spaces, is nuclear, then it is an (LN)-space.

In the above definitions and theorems, we can without loss of generality substitute Hilbert spaces $H_{n}$ for the Banach spaces $E_{n}$ and Hilbert-Schmidt mappings for nuclear mappings.

In what follows we need the concept of a reproducing kernel. We quote the definition of Aronszajn [1], [6]. Let $H$ be a Hilbert space with scalar product $(,)_{x}$, consisting of functions $f(x)$ defined on some point set $G$. The function $K(x, y), x \in G, y \in G$ is called a reproducing kernel if:

(1) for every fixed $y$ the function $K(x, y)$ of $x$ belongs to $H$,

(2) $K(x, y)$ has the reproducing property

$$
f(y)=(f(x), K(x, y))_{x} \quad \text { for all } f \in H .
$$

THEOREM 3. Let $H_{n}, n=1,2, \cdots$, be a sequence of Hilbert spaces with reproducing kernels $K_{n}(x, y)$, where the scalar product in $H_{n}$ is 
given by

$$
(\phi, \psi)_{n}=\int \phi(x)(\overline{\psi(x)})-d \sigma_{n}(x)
$$

the $\sigma_{n}$ being certain measures having their supports in some fixed set $G$ and fulfilling the further condition

$$
\left.(\phi, \phi)_{n} \geqq(\phi, \phi)_{n+1} \quad \text { for all } \phi \in H_{n} \quad \text { (i.e. } H_{n} \vec{\subset} H_{n+1}\right) .
$$

If, for every $m$, there exists an $n>m$ such that the condition

$$
\int K_{m}(x, x) d \sigma_{n}(x)<\infty
$$

holds, then the space

$$
E=\underset{\rightarrow n}{\operatorname{ind}} H_{n}
$$

is an $(\mathrm{LN})-$ space.

REMARK. Because of Theorem 1, $E$ is then a nuclear space. The corresponding theorem for projective limit (1), is also true: in that case the condition (K) takes the form

$\left(\mathrm{K}^{\prime}\right) \quad \int K_{\beta(\alpha)}(x, x) d \sigma_{\alpha}(x)<\infty$, where $\alpha, \beta \in A$ and $\beta(\alpha)>\alpha$.

In preparation for using the above theorems to establish nuclearity for spaces of holomorphic functions, let us consider the Hilbert space $H_{0}=\left\{\phi \mid \phi(z)\right.$ holomorphic in $\left.G,\|\phi\|^{2}=\iint_{G}|\phi(z)|^{2}|g(z)|^{2} d z<\infty\right\}$. Here $G$ is some open set of the complex plane and $g(z)$ is some continuous (weight) function on $G$ different from zero. It can be shown that $H_{0}$ possesses a reproducing kernel $K_{0}(z, w)$, continuous on $G$ (this is a corollary of Hartogs' theorem), and satisfying the inequality

$$
K_{o}(w, w) \leqq \frac{1}{\left(\pi r^{2}\right)^{2}} \iint_{C(w, r)}|g(z)|^{-2} d z
$$

for every disc $C(w, r)$ contained by $G$.

Now let $G_{n}$ be a sequence of open sets (bounded or not) in the complex plane such that

$$
G_{n} \supset G_{n+1}, \quad n=1,2, \cdots,
$$

and let $g_{n}, g_{n}^{\prime}$ be continuous (weight) functions $\neq 0$, defined on $G_{1}$. Let us take the Hilbert spaces $H_{n}=\left\{\phi \mid \phi(z)\right.$ holomorphic on $G_{n}$, $\left.\|\phi\|_{H_{n}}^{2}=\iint G_{n}|\phi|^{2}\left|g_{n}\right|^{2} d z<\infty\right\}$, and the Banach spaces 


$$
M_{n}=\left\{\phi \mid \phi(z) \text { holomorphic on } G_{n},\|\phi\|_{m_{n}}^{2}=\sup _{z \in G_{n}}\left|\phi(z) g_{n}^{\prime}(z)\right|<\infty\right\} \text {. }
$$

We assume that the functions $g_{n}, g_{n}{ }^{\prime}$ are such that

$$
\begin{array}{ll}
H_{n} \vec{\subset} H_{n+1}, & n=1,2, \cdots, \\
M_{n} \vec{\subset} M_{n+1}, & n=1,2, \cdots
\end{array}
$$

Beside this we require $g_{n}, g_{n}^{\prime}$ to have the following properties:

For any $n$ there exists an $m(n)$ such that

$$
\text { 1. } m(n) \rightarrow \infty \text { if } n \rightarrow \infty, m<\infty \text {. }
$$

$$
\text { 2. } \iint_{G_{m}}\left|\frac{g_{n}}{g_{m}^{\prime}}\right|^{2} d z=A<\infty \text {. }
$$

For any $t \in G_{n}$ it is possible to find $d_{t}>0$ such that

$$
\text { 1. } C\left(t, d_{t}\right) \subset G_{m}
$$

$$
\text { 2. } \frac{\left|g_{n}^{\prime}(t)\right|}{\pi d_{t}^{2}}\left[\iint_{C\left(t, d_{t}\right)}\left|g_{m}(z)\right|^{-2} d z\right]^{1 / 2} \leqq B<\infty \text {, }
$$

hold for all $t \in G_{n}$ ( $n$ and $m$ as in $\left(\mathrm{N}_{1}\right)$ ). We can now state

THEOREM 4. If the conditions $\left(\mathrm{N}_{1}\right)$ and $\left(\mathrm{N}_{2}\right)$ are fulfilled, the equivalence

$$
E=\operatorname{ind}_{\rightarrow n} H_{n} \cong \text { ind } M_{n}
$$

holds, and $E$ is an (LN)-space.

REMARK. In proving the nuclearity we use essentially inequality (I).

If for every $n, g_{n}=g_{n}{ }^{\prime}$, these functions being holomorphic, and if the distances $d\left(G_{n+1}, C G_{n}\right)$ are all positive (when $C G_{n}=\varnothing, d\left(G_{n+1}, C G_{n}\right)$ is positive by convention) Theorem 4 follows from the single condition $\left(\mathrm{N}_{1}\right)$.

The corresponding theorem for projective limits (1) also holds under the following assumptions:

$$
G_{\alpha} \subset G_{\beta}, H_{\beta} \vec{\subset} H_{\alpha}, M_{\beta} \vec{\subset} M_{\alpha}, \text { for } \alpha<\beta
$$

(the definitions of $H_{\alpha}, M_{\alpha}$ are analogous to those of $H_{n}$ and $M_{n}$ )

$$
g_{\alpha}, g_{\alpha}^{\prime} \text { continuous and } \neq 0 \text { on } \bigcup_{\alpha} G_{\alpha}
$$


$\left(\mathrm{N}_{1}^{\prime}\right) \quad \iint_{G_{\beta}}\left|\frac{g_{\alpha}}{g_{\beta}^{\prime}}\right|^{2} d z=A<\infty \quad$ for all $\alpha \in A$ and some $\beta(\alpha)>\alpha$,

1. $C\left(t, d_{t}\right) \subset G_{\beta}$,

$\left(\mathrm{N}_{2}^{\prime}\right)$

$$
\text { 2. } \frac{\left|g_{\alpha}^{\prime}(t)\right|}{\pi d_{t}^{2}}\left[\iint_{C\left(t, d_{t}\right)}\left|g_{\beta}\right|^{-2} d z\right]^{1 / 2} \leqq B<\infty \text {, }
$$

for some $d_{t}>0$ and all $t \in G_{\alpha}$.

The above theorems can be used to obtain information about the structure of Gelfand's $W$-and (E-distribution spaces, and to prove their nuclearity (for the definitions of these spaces, see [7] and [11]). These theorems also imply that Silva's ultradistribution spaces [9], and the boundary distribution spaces of Köthe [5] and Tillmann [10] are nuclear. As still another application, one can obtain simple proofs for the nuclearity of the spaces $S(G)$ considered by Grothendieck [3] and Köthe [4].

Proofs of these and other results will appear in [12].

\section{BIBLIOGRAPHY}

1. N. Aronszajn, Theory of reproducing kernels, Trans. Amer. Math. Soc. 68 (1950), 337-404.

2. A. Grothendieck, Produits tensoriels topologiques et espaces nucléaires, Mem. Amer. Math. Soc. No. 16 (1955).

3. - Sur certains espaces de fonctions holomorphes. I, II, J. Reine Angew. Math. 192 (1953), 35-64, 77-95.

4. G. Köthe, Dualität in der Funktionen-theorie, J. Reine Angew. Math. 191 (1953), $30-49$.

5. — Die Randverteilungen analytischer Funktionen, Math. Z. 57 (1952), 13-33.

6. H. Meschkowski, Hilbertsche Räume mit Kernfunktion, Springer-Verlag, BerlinGöttingen-Heidelberg, 1962.

7. W. P. Palamodow, The Fourier transformation of quickly increasing infinitely differentiable functions, Trudy Moskov. Mat. Obšč. 11 (1962), 309-350. (Russian)

8. D. A. Rałkow, On a certain property of nuclear spaces, Uspehi Mat. Nauk 12 (1957), 231-236. (Russian)

9. J. Sebastião e Silva, Les fonctions analytiques comme ultra-distributions dans le calcul opérationnel, Math. Ann. 136 (1958), 58-96.

10. H. G. Tillmann, Randverteilungen analytischer Funktionen und Distributionen, Math. Z. 59 (1953), 61-83.

11. J. Wloka, Über die Hurewicz-Hörmanderschen Distributionsräume, Math. Ann. (to appear).

12. - Reproduzierende Kerne und nukleare Räume, Math. Ann. (to appear).

University of California, Los Angeles and

UNIVERSITY OF HEIDELBERG, GERMANY 